den Walen. Auf dem Bugrand ihres Kanus stehend, harpunierten sie ihre Beute. Die Fisch-, Schildkröten- und Seekuhnahrung ergänzten sie mit den Eiern von Schildkröten und Vögeln, welche sie auf den vor der Küste liegenden Riffen und Inseln sammelten. Aber auch alle möglichen Pflanzen und Pflanzenteile gehörten zur Mahlzeit. Bei stürmischem Wetter suchten die Frauen in der nahen, geschützten Flußmündung nach Schaltieren. Sie sammelten Pflanzen, zum Beispiel Jamwurzeln, die im feuchten Dschungel in der Nähe der Küste wild gediehen, und auch manche Früchte und die Eier von Buschhühnern und Busch-Tale-Galahühnern (Großfußhühner oder Wallnister), je nach der Jahreszeit.

All dies erkannte ich nicht sogleich. Es war ein Teil der Erfahrungen, die ich unter diesen Eingeborenen sammelte, als ich nach und nach ihre Freundschaft gewann und in ihre Lager aufgenommen wurde und mich unter ihnen niederlie $\beta$, um wie sie zu leben. So begannen die langen Jahre, die ich unter den letzten nomadischen Jägern von Cape York und anderen Gebieten Australiens verbrachte.

\title{
SHOWA-SHINZAN \\ Der jüngste Vulkan im südlichen Hokkaido, Japan
}

OSKAR BÄR

Die Geburt des Vulkans Paracutin in Mexico fand bei uns große Beachtung. Es erschienen verschiedene Publikationen darüber, und ein ausgezeichneter Schulfilm (SAFU 3o1) entstand.

In unseren Geographenkreisen recht unbemerkt verlief hingegen die nicht minder faszinierende Eruption des Usu-Vulkans auf Hokkaido in den Jahren 1943 bis 1945. Sie führte zur Bildung des Shôwa-Shinzan. Das japanische Wort «Shinzan» bedeutet «neuer Berg» (san = Berg; z. B. Fuji-san). "Shôwa» ist der Name des heutigen Kaiserhauses (1926- -000 ). "Shôwa-Shinzan» besagt folglich: neuer Berg der Shôwa-Aera. Desgleichen heißt ein 1910 entstandener Vulkankegel der gleichen Region Meiji-Shinzan (Meiji-Aera: 1868-1912).

Auf einer zehntägigen Exkursion ins Vulkangebiet Süd-Hokkaidos im Frühjahr 1964 hatte ich Gelegenheit, diese interessanten Erscheinungen zu studieren. Aus den Gesprächen mit Prof. Dr. T. Ishikawa, einem führenden japanischen Vulkanologen, der mich auf der Exkursion begleitete, und mit Hilfe seiner Publikationen habe ich diesen kurzen Bericht zusammengestellt. Herrn Prof. Ishikawa sei an dieser Stelle herzlich gedankt.

Der Vulkan Usu liegt etwa $30 \mathrm{~km}$ NNW der Stadt Muroran in Süd-Hokkaido, zwischen der Funka-Bay (Eruptions-Bucht) und dem Tôya-See (vulkanischer CalderaSee). Er bedeckt eine Fläche von rund $30 \mathrm{~km}^{2}$ (Durchmesser ca. $6 \mathrm{~km}$ ), und sein höchster Punkt, der Usu-Dom, erreicht 725 m über Meer.

Die vulkanische Tätigkeit begann beim Usu als Folge des Caldera-Einbruchs (zusammen mit der Entstehung des Tôya-Nakajima im Zentrum des Caldera-Sees) gegen Ende des Pleistozäns. Sie dauert bis heute an. Bekannte Eruptionen fanden in folgenden Jahren statt: 1663 (Kanbun Aera), 1768 (Meiwa Aera), 1822 (Bunsei Aera), 1853 (Kaei Aera) und 1910 (Meiji-Aera). Der Vulkan baut sich zur Hauptsache aus verschiedenen andesitischen Laven auf. Wichtig sind auch Aschenlagen und mächtige Bimssteinschichten, die oft deutlich erkennbare Plateaus bilden.

Der neuste Ausbruch im Jahre 1943 ereignete sich am Osthang des Hauptvulkans, etwa zwei Kilometer vom Usu-Dom entfernt, wenig westlich des Dorfes Fukaba. Der ganze Ablauf des Geschehens läßt sich in drei Abschnitte einteilen: 


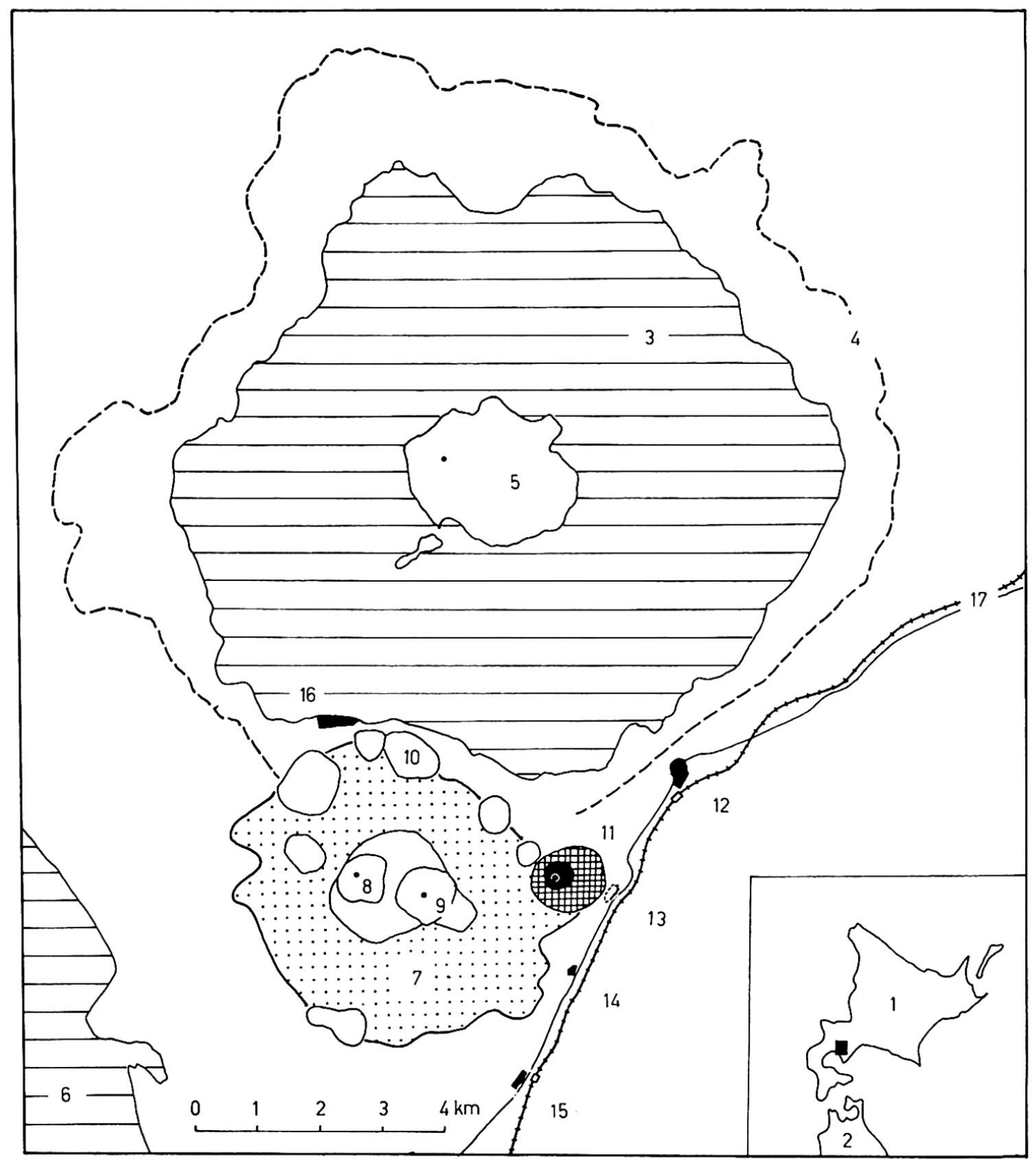

Abb. 1: Tôya-See und Usu-Vulkan.

$1=$ Hokkaido, $2=$ Honshu, $3=$ Tôya-See, $4=$ Calderarand, $5=$ Nakajima, $6=$ Funka-Bucht, $7=$ UsuVulkan, $8=$ Kousu-Dom, 9=Ousu-Dom, 10=Meiji-Shinzan, 11=Shôwa-Shinzan, 12=Sobetsu, 13= Fukaba (zerstört), 14=Yanagiwara, 15=Kamiosaru, 17= Bahnlinie und Straße.
A. Erdbebentätigkeit: 28. 12.1943 - 22.6.1944
B. Explosionsphase: 23.6. 1944 - 31. 10. 1944
C. Aufbau des Lavadoms: 1. 11. 1944-ca. Ende 9. 1945

A. Erdbebentätigkeit: Starke Erdbeben eröffneten diese Phase am 28. 12. 1943. Ihre Stärke und Zahl wuchs bis Ende des Jahres auf 200 täglich an. Dann trat rasch eine Beruhigung ein. Man nimmt an, daß in dieser Zeit das Magma durch den zentralen Krater auszubrechen drohte, sich dann aber gegen eine Schwächezone im Südosten hin bewegte. Dort begann sich der Boden nun zu heben, und Spalten sprangen auf. In den 


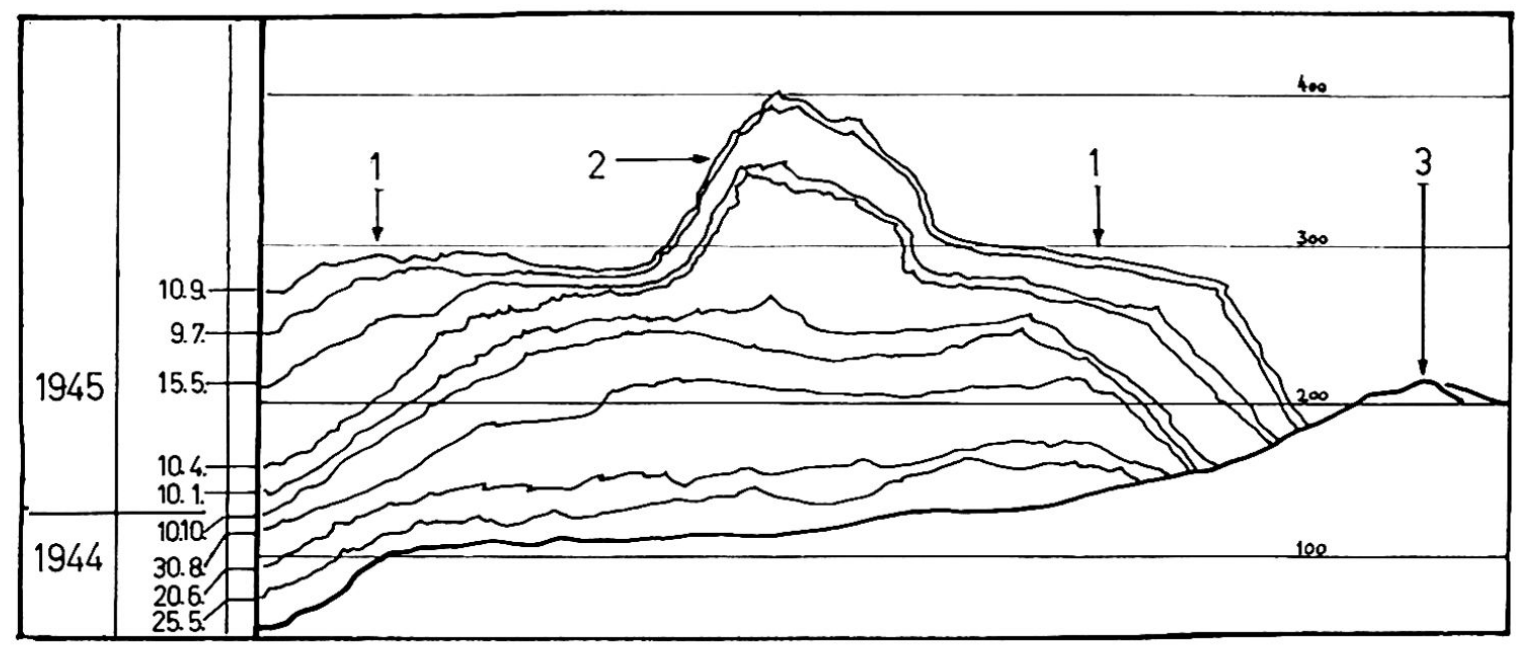

Abb. 2: Wachstumsdiagramm des Shôwa-Shinzan nach Mimatsu (vereinfacht). Nur die wichtigsten Phasen sind dargestellt. 1= "roof mountain», 2=Lavadom, 3=Erdoberfläche vor dem Ausbruch.

nahen Dörfern sank der Wasserspiegel in den Brunnen ab, verschiedene Quellen erhöhten aber gleichzeitig ihre Förderung. Es folgten Zerstörungen von Kleindämmen und Häusern. Bis anfangs April wurde der Boden längs der Nord-Süd-Straße (Yanagiwara-Sobetsu) um $16 \mathrm{~m}$ gehoben. Dann verlagerte sich das Hebungszentrum mehr nach Norden. Die Hebung erreichte $20-50 \mathrm{~cm}$ täglich. In einem Maisfeld bei Fukaba betrug sie rund $50 \mathrm{~m}$. Die Erdbebenhäufigkeit.stieg in jener Zone in kurzer Zeit von 100 auf 250 Beben pro Tag an.

B. Explosionsphase: Am 23.6.1944, morgens um $8.30 \mathrm{Uhr}$, beobachtete man in Maisfeldern westlich Fukaba die ersten Zeichen aufsteigenden Rauchs. Explosionen von Wasserdampf folgten. Sie entstanden in nicht allzugroßer Tiefe im Grundwasser, das vom aufsteigenden Magma erhitzt wurde. Später setzten heftigere, vom Gasdruck des Magmas herrührende Explosionen ein. Schwefelhaltige Asche wurde ausgeworfen, die sich in Schichten bis $30 \mathrm{~cm}$ Dicke auf die Felder legte und großen Schaden anrichtete. Bis 1000 m hoch stieg manchmal die Rauchsäule. Während der darauffolgenden gesteigerten Explosionstätigkeit bildeten sich sieben kleine Krater. Oft schossen zerstörende Glutwolken daraus hervor. Durch den Aufstieg des Magmas hervorgerufene Hebungen schritten fort. Durchschnittlich 500, bisweilen aber bis 1000 Erdbeben erschütterten nun die gefährdete Zone täglich. Schließlich bildete sich eine flache, domartige Erhebung von $0,5 \mathrm{~km}^{2}$ Oberfläche. Die Maisfelder auf ihrem Rücken waren von 130 bis 160 auf rund $300 \mathrm{~m}$ über Meer gehoben worden. Das Dorf Fukaba wurde durch Hebung und Pressung mehr oder weniger zerstört. Die Iburi-Bahnlinie am Ostfuß des Vulkans mußte mehr nach Osten verlegt werden.

C. Aufbau des Lavadoms: Nach dem 1.11. 1944 klang die Explosionstätigkeit plötzlich ab. Eine schwarze pyramidenförmige Lavamasse begann aufzusteigen. Sie überdeckte nach und nach die kleinen Krater und verformte sich in einen Dom mit steilerem Südhang. Aufsteigender Rauch verhüllte ihn meistens. Durch die ausdauernde Beobachtung Herrn Mimatsus, des damaligen Posthalters von Sobetsu $(2 \mathrm{~km} \mathrm{NE} \mathrm{Shôwa-Shin-}$ zan) sind wir über die Wachstumsphasen gut orientiert (Mimatsu: Tagebuch eines Berges; vgl. Abb. 2). Der Lava-Dom erreichte seine größte Höhe im September 1945 mit $404 \mathrm{~m}$ über Meer, $110 \mathrm{~m}$ höher als der sog. «roof mountain». Sein Durchmesser beträgt heute am Fuß etwa $300 \mathrm{~m}$. Auf seinem Rücken wurde eine Sedimentgesteinsdecke mit Felsbrocken und Schotterlagen des frühern Talbodens in die Höhe getragen. Im Kontakt mit den heißen Lavamassen entstanden dabei aus Lehm- und Tuffschichten 


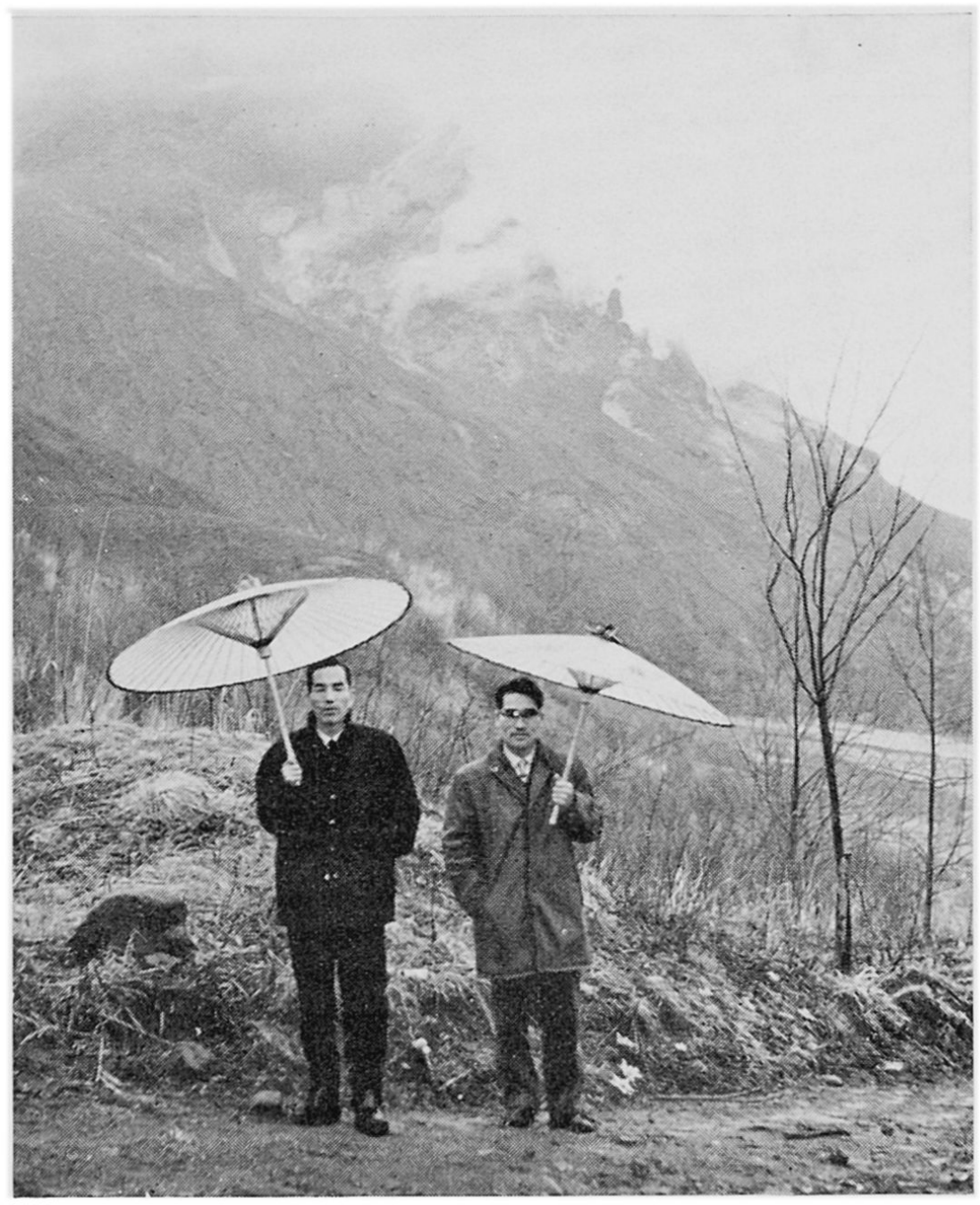

Abb. 3:

Rauchender Lavadom des Shôwa-Shinzan von Westen.

Betrachtungsstandort : roof mountain.

Aufnahme am

6. April 1964

natürlich gebrannte Ziegelsteine. Durch ticfe Spalten in diesem überdeckenden Material sah man da und dort die rotglühende Lava. Ihre Temperatur betrug im Maximum $980^{\circ}$. Am 18. 8. 1946 wurde der neue Lavadom erstmals bestiegen. Die Untersuchung des gesammelten Materials ergab Dazit, der vom Material der Nachbardome Ousu und Kousu nur wenig abweicht.

In den 18 Jahren seit 1946 ist die Temperatur des Lavadoms nur langsam gesunken. In den Spalten werden heute ca. $650^{\circ}$ gemessen. Auch die Fumarolentemperatur ist von $1000^{\circ}$ im Jahre 1947 beinahe linear auf $680^{\circ}$ im Jahre 1962 abgesunken (Diagramm von M. Seino). Immer noch quellen aus Spalten und Rissen graue Rauch- und Wasserdampfwolken. Sie umhüllen die bizarren rot-gelb-braunen Nadeln des Doms und scheinen ihm durch ihre mannigfaltigen Bewegungen das Leben zurückzugeben (Abb. 3 ). Wenn man in Betrachtung dieser Vorgänge auf dem «roof mountain» steht, kann man kaum erfassen, daß der scheinbar feste Grund, auf dem man vertrauensvoll seinen Fuß gesetzt hat, innerhalb weniger Monate um $150 \mathrm{~m}$ gehoben werden konnte. Es scheint kaum möglich, daß anstelle des urweltlichen Schauspiels, das sich darbietet, vor nicht langer Zeit japanische Bauern ihre Felder bestellten. Vulkanologen haben aber beide Zustände photographiert, und Herr Mimatsus Pinsel hat sie mit zarten Farben auf japanische Seide gezaubert (Abb. 4 und 5). 


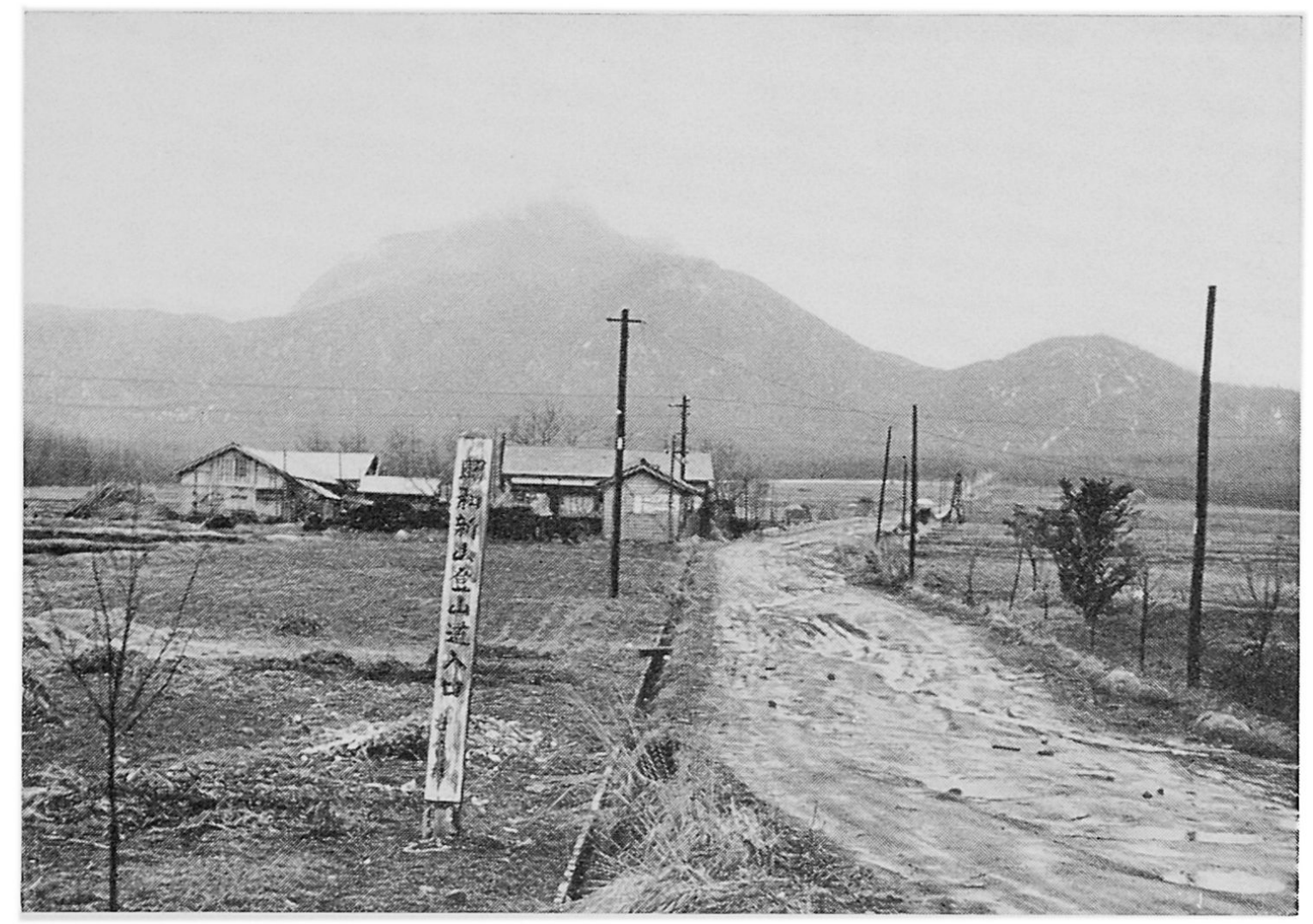

Abb. 4: Shôwa-Shinzan von Norden. Alluvialebene (150 m ü. Meer), roof-montain (300 m ü. Meer) und Lavadom (ca. $400 \mathrm{~m}$ ü. Meer) sind gut zu unterscheiden.

Aufnahme am 6. April 1964.

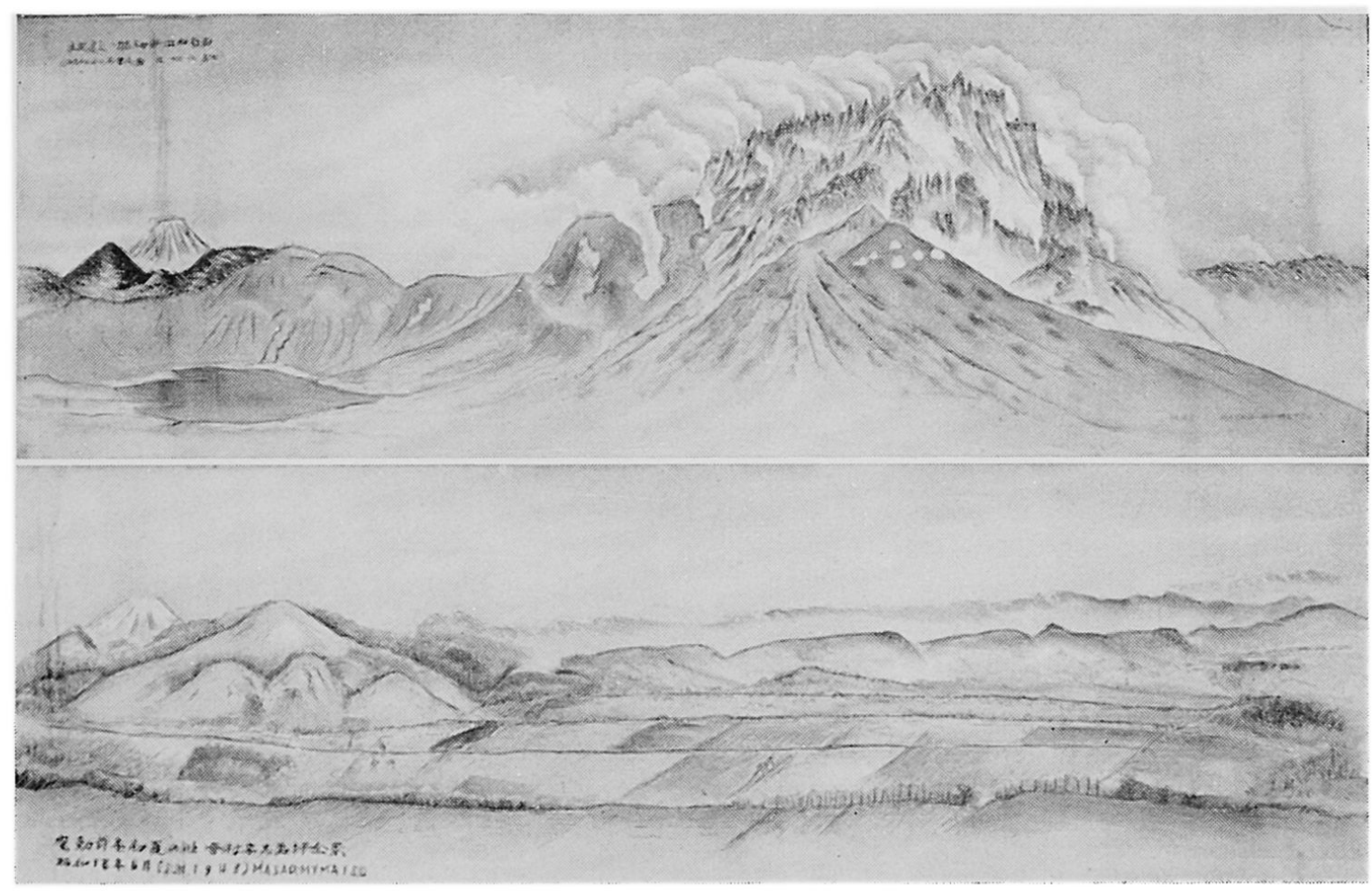

Abb. 5: Seidenmalerei, die Zustände vor (unten) und während des Ausbruchs (oben) darstellend. Herr Mimatsu hat beide Bilder am gleichen Standort (südlich) gemalt. Links hinten ist immer der Vulkan Yotei-san zu erkennen. Er wird wegen seiner idealen Form auch Jesso-Fuji (Jesso=Hokkaido) genannt. 


\section{SHOWA-SHINZAN \\ Youngest volcano in southern Hokkaido, Japan}

Usu-volcano (near Muroran in southern Hokkaido) is still an active volcanic zone. Five eruptions between 1663 and 1910 are well nown. The recent activity $(19+3-45)$ may be divided into three stages, which are typical for Usu-volcano.

1. Earthquake stage: $28.12 .43-22.6 .4+$

2. Explosion stage: $23.6 .44-31.10 .44$

3. Dome building stage: $1.11 .44-9.45$

The maximum height of the dome was $40+\mathrm{m}$ above sea-level in September 1945 (about $250 \mathrm{~m}$ of growth; see Fig. 2). "The new lava is rich in silica content, ressembling that of dacite or rhyodacite» (T. Jshikawa).

\section{DER \\ 20. INTERNATIONALE GEOGRAPHENKONGRESS IN LONDON \\ (20.-28. Juli I 964)}

WERNER KÜNDIG-STEINER

Zum zwanzigsten Mal strömten die Geographen Mitte Juli aus aller Welt zu einem Kongreß, diesmal in der wärmeüberfluteten Metropole London, zusammen. Rund 2100 eingeschriebene Teilnehmer belebten die im Neubau begriffene Technische Hochschule (Imperial College) sowie das Haus der «Royal Geographical Society». In 8 Sektionen und 17 Kommissionen kamen 450 Referate zur Diskussion, zudem fanden 5 Abendvorträge und 13 Ausstellungen, vor Kongreßbeginn überdies 20, nach dem Kongre $\beta 16$ Symposien bzw. Feldstudien statt und wurden rund 50 Exkursionen in und um London durchgeführt. Die naturgemäß umfangreichen Vorbereitungen besorgte ein 24-köpfiges Nationalkomitee, das der liebenswürdige Prof. Dudly Stamp präsidierte. Ihm standen rund ein Dutzend Arbeitsgruppen sowie während des Kongresses etwa 200 Studenten zur Verfügung. Auch der Außenstehende konnte, insbesondere anläßlich der Einschreibung, die viele auf eine harte Geduldsprobe stellte, ermessen, welches gewaltige Ausmaß an Kleinarbeit von den englischen Geographen gemeistert wurde. Auch bei andern Gelegenheiten zeigte sich indessen, daß die geographischen Weltkongresse mehr und mehr einen Umfang angenommen haben, der sich kaum mehr bewältigen läßt. Dabei war das Bedürfnis nach internationalem fachwissenschaftlichem Meinungsaustausch äußerst stark, so daß die Organisatoren künftiger Kongresse gerade diesem Punkte ein besonderes Augenmerk zu widmen haben werden.

Erwartungsgemäß stammte der Hauptharst, d.h. etwa $55 \%$ aller Teilnehmer aus Großbritannien und den USA. Sie bestimmten nicht nur sprachlich, sondern auch fachlich und ideell den Kongreß maßgeblich. Es wurde zu $88 \%$ in englischer und nur zu $12 \%$ in französischer Sprache vorgetragen, während vor gut 3 Jahrzehnten am Pariser Kongreß genau das umgekehrte Verhältnis geherrscht hatte. Die deutschen Teilnehmer fühlten sich insofern «benachteiligt», als ihre Kollegen aus der DDR in letzter Minute aus visumstechnischen Gründen ihren Besuch absagen mußten. Man vermißte aber auch manchen bekannten Geographen aus der Bundesrepublik. 